\title{
Study on the optical properties of selective emission materials by Finite-Difference Time-Domain Method
}

\author{
ZHAO Shuang, ZHANG Zhifang, PAN Xiaojie, ZHU Yanyan \\ Shanghai University of Electric Power, Shanghai 200090, China \\ e-mail: yyzhu@shiep.edu.cn
}

Key words: selective emission; Finite-Difference Time-Domain method; rare earth oxides

\begin{abstract}
The optical properties of one-dimensional photonic crystals with $\mathrm{Er}_{2} \mathrm{O}_{3} / \mathrm{Si}$ structure are designed and calculated by Finite-Difference Time-Domain method. The influence of different thickness and reflectivity on the radiation properties were studied. It was found that the radiation spectral intensity at high temperatures was drastically reduced near the wavelength of $1.8 \mu \mathrm{m}$ for a $40 \% \mathrm{Er}_{2} \mathrm{O}_{3}+60 \% \mathrm{Al}_{2} \mathrm{O}_{3}$ sample due to the strong oscillations of the reflectance.
\end{abstract}

\section{Introduction}

There is a surge of scientific interest in improving the efficiency of solar cells. Studies have shown that [1-3] there is a main factor that causes the energy loss of solar cells. The light absorbed by the solar cells whose energy are lower than its gap can not produce electron-hole pairs. In order to improve the efficiency of solar cells, the light absorption range of the battery may be expanded the infrared range. The thermal photovoltaic (TPV) power generation system absorbs infrared light from the emitter and converts it into electrical energy. It can provide stable energy regardless of the weather[3, 4].

Since the light of the thermal photovoltaic cells comes from the emitter, the luminous performance of the emitters directly affects the photoelectric conversion efficiency of the whole system. By improving the emissivity of the emitter and adjusting the emission spectrum, the main luminescence band can match that of the thermal photovoltaic cell. In this way, the efficiency of the thermal photovoltaic power generation system can be greatly improved[5]. The traditional thermal emitters are graphite, silicon carbide, silicon nitride and so on. Although the preparation of these existing materials is mature and luminous efficiency is very high, the radiation spectra of these materials belong to the gray body radiation whose distribution spectral is very wide. So the photoelectric conversion efficiency is not high. Recently, the concept of selective emitters has been proposed. The biggest difference between the selective emitter and the gray body emission is that the emission spectrum of selective emitter is mainly concentrated in one or several bands. If the response spectrum of the light-emitting and thermal PV cells can match well, high efficiency heat can be obtained for the photovoltaic power generation system [5].

Rare earth elements are active due to the $4 \mathrm{f}$ and $5 \mathrm{~s} / 5 \mathrm{p}$ electron orbital configurations, which can produce a wide variety of radiation absorption and emission. Rare earth material has a strong ability to absorb light and its emission spectrum is narrow. These advantages determine that it can be an emitter of a thermal PV system [6]. In 1972, Guazzoni et [7] reported high temperature emission spectrum of erbium, samarium, neodymium, ytterbium and other rare earth oxide ceramics [7]. Among them, $\mathrm{Er}_{2} \mathrm{O}_{3}$ is a kind of ideal emitter which has a high melting point, whose photon energy 
produced by electron transition is $0.805 \mathrm{eV}(1540 \mathrm{~nm})$ that match the gap of Ge (The band gap is $0.661 \mathrm{eV}$ ) and $\mathrm{GaSb}$ (The band gap is $0.726 \mathrm{eV}$ ). And, reports about physical properties of thermal photovoltaic cells for $\mathrm{Er}_{2} \mathrm{O}_{3}$ as selective emitter material is still quite few.

With the rapid development of micro-nanotechnology, it was found that when the microstructure of the material surface is close to the wavelength of light, this microstructure will affect the radiation characteristics of the material. Then, the selective radiator selective radiator with a periodic microstructures were proposed. The periodic microstructured selective radiator can be divided into one-dimensional, two-dimensional and three-dimensional ones. A typical one-dimensional microstructured selective heat radiator is made by stacking which are easy to combine with other devices, whose spectral control only rely on its band gap.

\section{Calculation method}

Finite-Difference Time-Domain (FDTD) was established in 1966 whose core idea is to convert the Maxwell's equation into a differential form, simulate the electron pulse and The time domain response of an ideal conductor. It is known as the most concerned and the most fashionable algorithm in the current electromagnetic field, but it also continue to develop and improve.

Finite-Difference Time-Domain (FDTD) method is to differentiate the Maxwell's equation in time and space. It can directly simulate the distribution of the field with high accuracy.

The microstructured selective radiator was made of two materials with different refractive index. So, $\mathrm{Si}$ and $\mathrm{Er}_{2} \mathrm{O}_{3}$ were chosen here. The gap is formed when radiation wave incident on this microstructure. The wavelength formula in the microstructure can be shown in Equation (1) and (2). [7]

$$
\begin{gathered}
\lambda_{1}=\frac{\pi\left(d_{h} n_{h}+d_{l} n_{l}\right)}{n_{h}-n_{l}} \\
\lambda_{2}=\frac{\pi\left(d_{h} \sqrt{n_{h}^{2}-1}+d_{l} \sqrt{n_{l}^{2}-1}\right)}{\arccos \left[\frac{n_{h}^{2} \sqrt{n_{l}^{2}-1}-n_{l}^{2} \sqrt{n_{h}^{2}-1}}{n_{h}^{2} \sqrt{n_{l}^{2}-1}+n_{l}^{2} \sqrt{n_{h}^{2}-1}}\right]} \\
\Delta g=\frac{2}{\pi} \arcsin \frac{n_{h}-n_{l}}{n_{h}+n_{l}}
\end{gathered}
$$

According to the above equations, the radiation in the range of $\lambda_{1} \sim \lambda_{2}$ will be reflected and will have multiple values. So the reflection band will have multiple wave ranges. As the denominator value becomes larger, the corresponding reflected band will move to the smaller wavelength region, and the distance between $\lambda_{1}$ and $\lambda_{2}$ will become smaller. According to formula (3), $\Delta g$ can be obtained.

\section{Results and discussion}

As the current widely used PV battery is GdSb battery, its forbidden band width is $0.72 \mathrm{eV}$, the 
corresponding wavelength is about 1.8 microns. After selecting two materials, $\mathrm{n}_{\mathrm{h}}$ and $\mathrm{n}_{1}$ are obtained. $\triangle \mathrm{g}, \lambda 0, \lambda 1$, and $\lambda 2$ are achieved. In this paper, Si films and $\mathrm{Er}_{2} \mathrm{O}_{3}$ films were used to simulate. The refractive index of the $\mathrm{Si}$ films was 3.42, the refractive index of $\mathrm{Er}_{2} \mathrm{O}_{3}$ films was 2.05 [8], the corresponding film thickness $d_{h}$ and the $d_{l}$ were $152.78 \mathrm{~nm}$ and $254.89 \mathrm{~nm}$. Taking these parameters into the above formula, we get the corresponding main bandage of 1.8 to 2.5 microns. The transmittance of $\mathrm{Si} / \mathrm{Er}_{2} \mathrm{O}_{3}$ is calculated by FDTD method when the vertical incidence of $\mathrm{Si}_{2} / \mathrm{Er}_{2} \mathrm{O}_{3}$ is five cycles which is shown in Fig. 1.

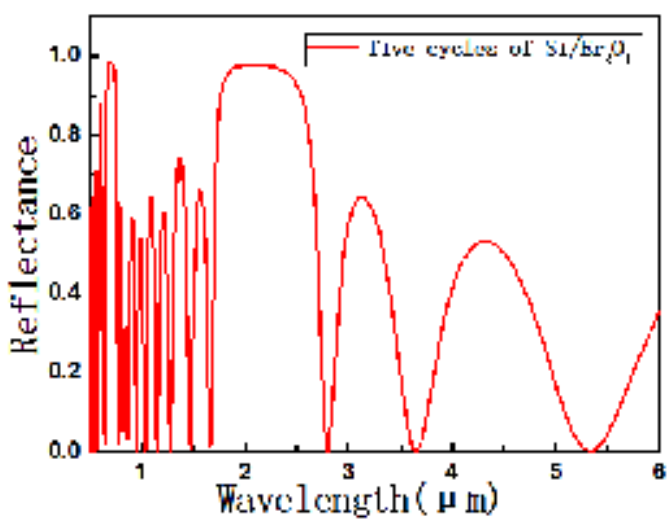

Fig.1 the reflectivity for 5 cycles of $\mathrm{Si} / \mathrm{Er}_{2} \mathrm{O}_{3}$ structures

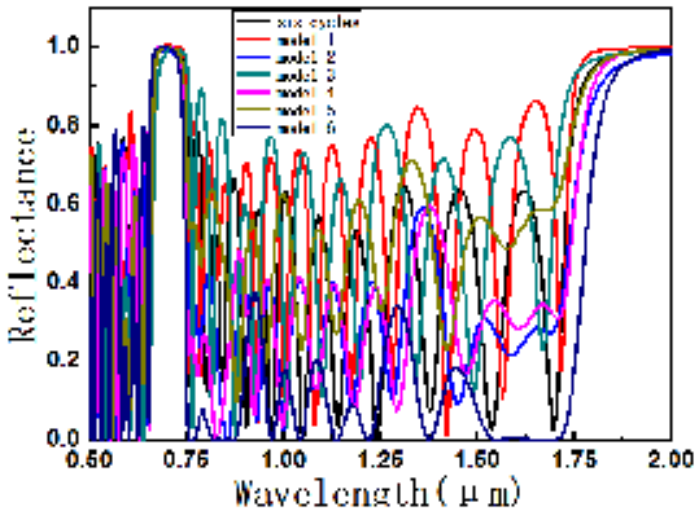

Fig.2 the effect of different cycles on the gap.

It can be seen from Figure 1 that the reflectivity in range of 1.8 to 2.5 microns is above 0.94 , which indicate that the structure forms a gap this range of 1.8 to 2.5 microns, and the reflected wave interference with the surface of the microstructure .

Figure 2 shows the effect the four, five, six, and seven cycles of the $\mathrm{Si} / \mathrm{Er}_{2} \mathrm{O}_{3}$ microstructures on the gap. The reflectivity is close to 1 in the gap band when the number of cycles reaches six. The more the number of cycles causes the more oscillations in the nonblocking zone. The number of different cycles does not affect the position of the band, which is consistent with the number of cycles in the above formula for calculating the band. It is known that to form a high reflective interference needs a sufficient number of cycle structure. And the more the cycle numbers the more the difficulty in the process of actual preparation. According to the result shown in figure 2, the sixth cycle meets the requirements. So in the following analysis it will be based on the six cycle structures.

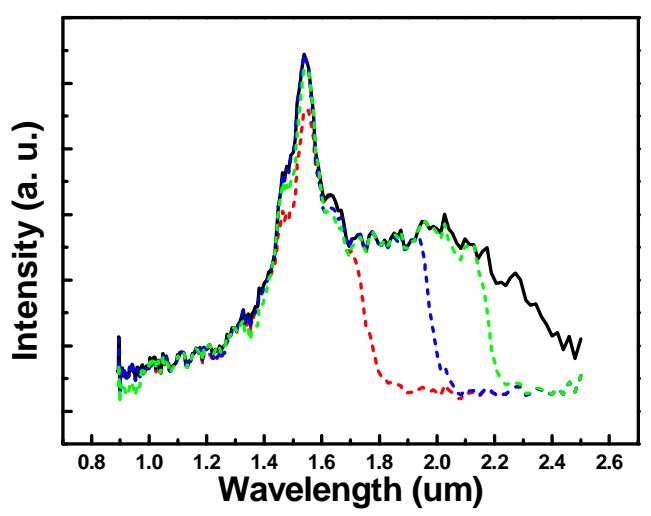

Fig. 3 Theradiation spectrum at $750^{\circ} \mathrm{C}$. The black solid line is the experimental data for the sample of the $40 \% \mathrm{Er}_{2} \mathrm{O}_{3}+60 \% \mathrm{Al}_{2} \mathrm{O}_{3}$ and the other three dashed lines are calculated one for the sample of the $40 \% \mathrm{Er}_{2} \mathrm{O}_{3}+60 \% \mathrm{Al}_{2} \mathrm{O}_{3}$ with $0.5 \mathrm{Er}_{2} \mathrm{O}_{3}+6$ cycles $-0.5 \mathrm{Er}_{2} \mathrm{O}_{3}$ microstructures. 
Figure 3 shows the radiation spectrum at $750^{\circ} \mathrm{C}$. The black solid line is the experimental data for the sample of the $40 \% \mathrm{Er}_{2} \mathrm{O}_{3}+60 \% \mathrm{Al}_{2} \mathrm{O}_{3}$ and the other three dashed lines are calculated one for the sample of the $40 \% \mathrm{Er}_{2} \mathrm{O}_{3}+60 \% \mathrm{Al}_{2} \mathrm{O}_{3}$ with $0.5 \mathrm{Er}_{2} \mathrm{O}_{3}+6$ cycles $-0.5 \mathrm{Er}_{2} \mathrm{O}_{3}$ microstructures. From the figure it is found that the there is a clear cut-off area at $1.8 \mu \mathrm{m}, 2.0 \mu \mathrm{m}, 2.2 \mu \mathrm{m}$ if the samples combined withmicrostructures. Therefore, the microstructures can reduce the radiation outside the characteristic wavelength.

\section{Conclusions}

In conclusion, by Finite-Difference Time-Domain method, the optical properties of one-dimensional photonic crystals with $\mathrm{Er}_{2} \mathrm{O}_{3} / \mathrm{Si}$ structure are designed and calculated. It was found that the radiation spectral intensity at high temperatures was drastically reduced near the wavelength of $1.8 \mu \mathrm{m}$ for a $40 \% \mathrm{Er}_{2} \mathrm{O}_{3}+60 \% \mathrm{Al}_{2} \mathrm{O}_{3}$ sample due to the strong oscillations of the reflectance in the microstructures.

\section{Acknowledgements}

This work was supported by the National Natural Science Foundation of China (51672172), Shanghai Natural Science Foundation(15ZR1418700).

\section{References}

[1] D. L. Chubb, and R. A. Lowe, J. Appl. Phys. 74 (9), 5687, 1993.

[2] C. M. Wang, Y. C. Chang, M. W. Tsai, Y.H. Ye, C. Y. Chen, Y. W. Jiang, Y. T. Chang, S. C. Lee, and D. P. Tsai, Optics Express, 15(22), 14673, 2007.

[3] D. Kraemer, L. Hu, A. Muto, X. Chen, G. Chen, and M. Chiesa, Appl. Phys. Lett. 92, 243503, 2008.

[4] K.R. Catchpole , K.L. Lin, M.A. Green, A.G. Aberle, R. Corkish, J. Zhao, and A. Wang, Solar Energy 76, 251, 2004.

[5] T. J. Coutts, Renewable and Sustainable Energy Reviews, 3, 77, 1999.

[6] D. Diso , A. Licciulli, A. Bianco , M. Lomascolo, G. Leo , M. Mazzer, S. Tundo, G. Torsello, and A. Maffezzoli, Materials Science and Engineering B 98, 1442003.

[7]Nagpal, P., Han, S.E., Stein, A. and Norris, D.J. Efficient Low-Temperature Thermophotovoltaic Emitters from Metallic Photonic Crystals. [J]. Nano Letters, 2008, 8, 3238-3243.

[8]Palik E D. Handbook of Optical Constants of Solids. New York: Academic Press Inc. 1985. 